\title{
Research Paper p63 $\alpha$ modulates c-Myc activity via direct interaction and regulation of MM1 protein stability
}

\author{
Anning Han ${ }^{1}$, Juan $\mathrm{Li}^{1, *}$, Yimin $\mathrm{Li}^{1,}{ }^{*}$, Yang Wang ${ }^{1}$, Johann Bergholz ${ }^{1}$, Yujun Zhang ${ }^{1}$, \\ Chenghua $\mathrm{Li}^{1}$, Zhi-Xiong Xiao ${ }^{1}$ \\ ${ }^{1}$ Center of Growth, Metabolism and Aging, Key Laboratory of Biological Resources and Ecological Environment of Ministry of \\ Education, College of Life Sciences, Sichuan University, Chengdu 610064, China \\ "These authors have contributed equally to this work \\ Correspondence to: Chenghua Li, email: lichenghua@scu.edu.cn \\ Zhi-Xiong Xiao, email: jimzx@scu.edu.cn
}

Keywords: p63, MM1, c-Myc, protein stability, cell cycle

Received: January 07, $2016 \quad$ Accepted: May 16, 2016

Published: June 20, 2016

\begin{abstract}
Both p53-related p63 and c-Myc are transcription factors playing key roles in cell proliferation, survival, development and tumorigenesis. In the present study, we identified that MM1, a c-Myc inhibitor, specifically binds to C-termini of p63 $\alpha$ (including $\Delta N p 63 \alpha$ and TAp63 $\alpha$ ). Further study demonstrates that p63 $\alpha$ facilitates MM1 protein degradation via proteasomal pathway, resulting in elevation of c-Myc transactivation activity. Knockdown of $\Delta \mathrm{Np} 63 \alpha$ leads to decrease in c-Myc protein levels, concomitant with reduced expression of CDK4 and Cyclin D1, and impaired cell cycle progression, both of which are effectively reversed by simultaneous knockdown of MM1. Moreover, expression of p63 and CDK4 is concomitantly up-regulated in B-cell acute lymphoblastic leukemia. Together, this study reveals a novel crosstalk between p63 and c-Myc that may play an important role in cell cycle progression and tumorigenesis.
\end{abstract}

\section{INTRODUCTION}

TP63, also known as the p63 gene, is a member of the p53 family, which locates on human chromosome $3 q 27-29[1,2]$. Due to usage of alternative promoters, the p63 gene encodes two major classes of protein isoforms, TAp63 and $\Delta \mathrm{Np} 63$. TAp63 proteins contain a full-length transactivation domain (TAD) at their $\mathrm{N}$-termini that is homologous to that of p53, whereas $\Delta \mathrm{Np} 63$ proteins possess incomplete $\mathrm{N}$-terminal TADs. Owing to alternative splicing at the C-termini, TA or $\Delta \mathrm{N}$ class of p63 can generate at least five different isoforms, including $\alpha, \beta, \gamma, \delta$, and $\varepsilon$ [3]. p $63 \alpha$ proteins, including TAp $63 \alpha$ and $\Delta \mathrm{Np} 63 \alpha$, contain two unique domains at the C-termini, namely, a sterile alpha motif (SAM) involved in protein-protein interaction and a transactivationinhibitory domain (TID) [4]. However, each and every p63 isoform contains the same DNA binding domain (DBD) and oligomerization domain (OLD). It is known that $\Delta \mathrm{Np} 63 \alpha$ is the predominant isoform of p63 expressed in epithelial cells. Studies have shown that p63 proteins can function as transcription factors, controlling transcription of downstream genes, including p21, Bax, Puma, Dicer, MKP3, and genes involved in cell adhesion [5]. Consequently, p63 proteins play a key role in cell proliferation, survival, development and tumorigenesis [6].

c-Myc is an important transcription factor involved in cell proliferation, survival, differentiation and tumorigenesis. c-Myc modulates about $15 \%$ genes in organisms ranging from Drosophila to human, and is activated in about $20 \%$ malignant tumors [7, 8]. Downstream target genes of c-Myc include CDK4 [9, 10], Cyclin D1 [11], EZH2 [12], ECA39 [13, 14], eIF4E [15, 16], eIF-2 $\alpha$ [15], and cdc25A [16].

MM1 (Myc modulator 1), also known as prefoldin subunit 5 (PFDN5), is one of six subunits of prefoldin, a molecular chaperone complex that binds and stabilizes newly synthesized polypeptides [17-20]. MM1 has been reported to bind to $\mathrm{N}$-terminal transactivation domain of c-Myc (myc box 2) and represses c-Myc transactivity [17].

In this study, we report that p63a physically interacts with and destabilizes MM1, resulting in elevation of c-Myc transactivation activity. 


\section{RESULTS}

\section{MM1 binds to C-terminus of p63a}

As shown in Figure 1A, p63 $\alpha$ contains unique SAM and TID domains at the C-terminus (p63 $\alpha \mathrm{CT}$ ), which are involved in protein-protein interaction and transactivation inhibition [21]. To identify proteins that interact with this region, we employed a yeast two-hybrid system to screen a human mammary gland cDNA library. As listed in Supplementary Table 2, 14 different proteins were found to associate with p63 $\alpha \mathrm{CT}$, one of which was MM1. Since MM1 was previously reported to inhibit c-Myc, which is involved in cell cycle regulation, similar biological function that $\mathrm{p} 63 \alpha$ possesses, we focused on MM1 and $\mathrm{p} 63 \alpha$ in this study.

To confirm the interaction between MM1 and C-terminus of p63 $\alpha$ in mammalian cells, we employed coimmunoprecipitation (co-IP) experiments using H1299 cells transiently co-overexpressing MM1 and different p63 isoforms. As shown in Figure $1 \mathrm{~B}$ and $1 \mathrm{C}$ ), $\Delta \mathrm{Np} 63 \alpha$, but not $\Delta \mathrm{Np} 63 \gamma$, interacted with MM1. Further study revealed that endogenous $\Delta \mathrm{Np} 63 \alpha$ and MM1 proteins formed stable complexes in MCF10A cells (Figure 1D and 1E).

\section{Expression of p63 $\alpha$ down-regulates MM1 protein levels}

We previously reported that peptidyl-prolyl isomerase Pin 1 directly binds to and stabilizes p63 $\alpha$ protein [22]. To investigate whether MM1 can influence $\triangle \mathrm{Np} 63 \alpha$ protein levels, we co-expressed MM1 and $\Delta \mathrm{Np} 63 \alpha$ in H1299 cells. As shown in Figure 2A, expression of either $\Delta \mathrm{Np} 63 \alpha$ or TAp63 $\alpha$ significantly decreased protein level of MM1, but not GFP. By contrast, $\Delta \mathrm{Np} 63 \gamma$ failed to affect MM1. Further investigation reveals that ectopic expression of $\Delta \mathrm{Np} 63 \alpha$ resulted in significant decrease of endogenous MM1 protein levels in Hs5787T and A549 cells (Figure 2B).

We next knocked down endogenous $\Delta \mathrm{Np} 63 \alpha$ in MCF10A cells, which are non-transformed human breast epithelial cells predominantly expressing $\Delta \mathrm{Np} 63 \alpha$ isoform, using lentiviral-based short hairpin RNAs (shRNAs). As shown in Figure 2C, two different shRNAs against pan p63 effectively knocked down endogenous $\Delta \mathrm{Np} 63 \alpha$, resulting in significant increase of endogenous MM1. In addition, acute depletion of $\Delta \mathrm{Np} 63 \alpha$ led to decrease in c-Myc protein levels (Figure 2C), consistent with a previous report that p63 is necessary for proper expression of c-Myc via Wnt/ $\beta$-catenin and Notch pathways in human keratinocytes [23].

To investigate whether TAp63 $\alpha$ has the same effects to MM1 and c-Myc as that of $\Delta \mathrm{Np} 63 \alpha$, we used a human pancreatic cancer cell line, MiaPaCa-2, which predominantly expresses TAp63 $\alpha$ isoform [24]. As shown in Figure 2D, knockdown of TAp63 $\alpha$ in MiaPaCa cells led to a significant increase in MM1 and decreases in c-Myc protein levels. These data indicate that both TAp $63 \alpha$ and $\Delta \mathrm{Np} 63 \alpha$ isoforms can down-regulate MM1 protein expression.

\section{$\Delta \mathrm{Np63a}$ promotes MM1 proteasome-dependent degradation}

To examine whether $\Delta \mathrm{Np} 63 \alpha$-mediated reduction of MM1 protein is due to altered protein stability, we examined the effects of $\Delta \mathrm{Np} 63 \alpha$ on co-expressed MM1 in H1299 cells. The MM1 protein half-life was measured with cycloheximide (CHX) chase assay. As shown in Figure 3A and 3B, the MM1 protein half-life was significantly shortened when co-expressed with $\Delta \mathrm{Np} 63 \alpha$. Similarly, overexpression of $\Delta \mathrm{Np} 63 \alpha$ also decreased halflife of endogenous MM1 protein in H1299 cells (Figure 3C and 3D). Conversely, knockdown of endogenous $\Delta \mathrm{Np} 63 \alpha$ in MCF10A cells extended half-life of endogenous MM1 protein (Figure 3E and 3F). Furthermore, Q-PCR analyses indicated that MM1 mRNA levels were comparable in MCF10A cells with or without depletion of endogenous $\Delta \mathrm{Np} 63 \alpha$ (Figure $3 \mathrm{G}$ ). These data demonstrate that $\Delta \mathrm{Np} 63 \alpha$ promotes MM1 protein degradation.

To determine whether $\Delta \mathrm{Np} 63 \alpha$ destabilizes MM1 through proteasome, we used MG132 to inhibit proteasome in H1299 cells co-transfected with MM1 and $\Delta \mathrm{Np} 63 \alpha$. As shown in Figure $3 \mathrm{H}, \mathrm{p} 21^{\text {cip } 1}$, which is mainly degraded through the proteasomal pathway [25], was dramatically increased by treatment with MG132, indicating the effectiveness of MG132; MG132 significantly blocked $\Delta \mathrm{Np} 63 \alpha$-mediated MM1 protein degradation. These data suggest that $\Delta \mathrm{Np} 63 \alpha$ promotes proteasome-dependent degradation of MM1 protein.

\section{Ablation of $\Delta \mathrm{Np} 63 \alpha$ down regulates c-Myc transactivity and impair cell cycle progression}

c-Myc is a transcription factor which recognizes and binds to the E-box sequences in gene promoters and activates their transcription [26-28]. It's reported that MM1 represses c-Myc-mediated E-box-dependent transcription [20]. To test whether $\Delta \mathrm{Np} 63 \alpha$-mediated down-regulation of MM1 increases c-Myc-mediated transactivation, we performed a set of luciferase assays, using the MBC1-4-Luc reporter containing 4 E-box promoter regions of cyclin-dependent kinase 4 (CDK4) gene, which is a transcriptional target of c-Myc [9]. As shown in Figure 4A, c-Myc activated the luciferase expression of MBC1-4-Luc, which was suppressed by MM1, whereas $\triangle \mathrm{Np} 63 \alpha$ significantly rescues this MM1mediated repression of MBC1-4-Luc. These data suggest that $\Delta \mathrm{Np} 63 \alpha$ stimulates c-Myc-mediated transactivation via down-regulating MM1.

To investigate the influence of endogenous $\Delta \mathrm{Np} 63 \alpha$ and MM1 on c-Myc transactivity, we knocked down p63 $\alpha$ or (and) MM1 in MCF10A cells with lentiviral- 
A.

\begin{tabular}{|c|c|c|c|c|c|}
\hline$\Delta$ Np63a & & DBD & OLD & SAM & TID \\
\hline TAp63 $\alpha$ & TAD & DBD & OLD & SAM & TID \\
\hline$\Delta \mathrm{Np63 \alpha}$ & & DBD & OLD & & \\
\hline
\end{tabular}

B.
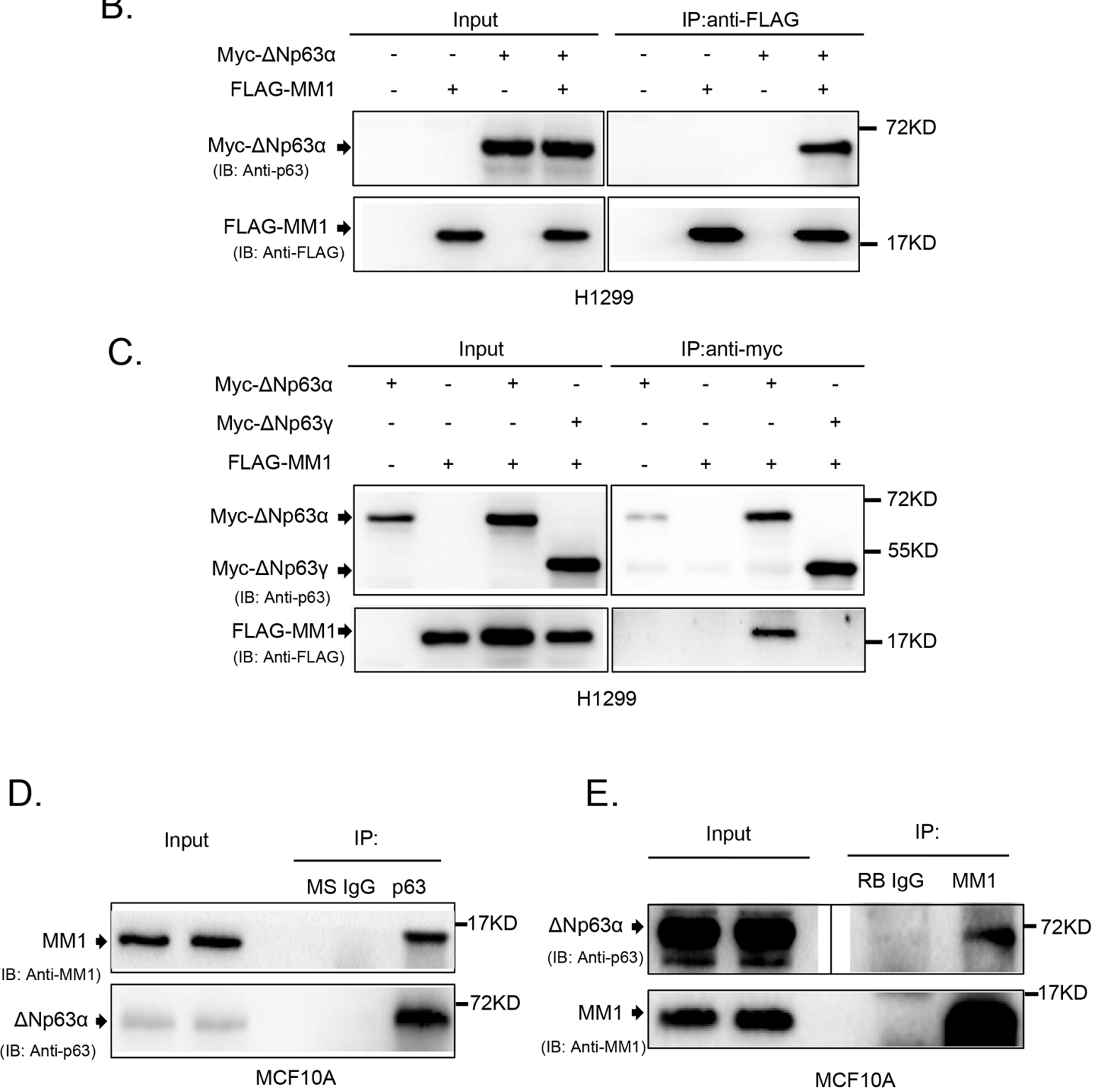

Figure 1: MM1 binds to C-terminus of p63a. A. Schematic structure of p63 protein isoforms. TAD: transactivation domain; DBD: DNA-binding domain; OLD: oligomerization domain; SAM: sterile alpha motif; TID: transinhibitory domain. The C-terminal fragment of p63 $\alpha$ (p63 $\alpha \mathrm{CT}$ ), containing SAM and TID regions, was cloned as a "bait" for yeast two-hybrid screening. B. and C. $\Delta \mathrm{Np} 63 \alpha$, but not $\Delta \mathrm{Np} 63 \gamma$, binds to MM1 in transfected cells. H1299 cells were co-transfected to express Myc-tagged $\Delta$ Np63 $\alpha$ or $\Delta$ Np63 $\gamma$ plus FLAG-tagged MM1 proteins. Cell lysates were subjected to immunoprecipitation (IP) with antibody to FLAG or Myc tag. The immunoprecipitates were analyzed by immunoblotting (IB) with anti-p63 and anti-FLAG. Whole cell lysates were used as input controls. D. and E. Endogenous proteins of $\triangle \mathrm{Np} 63 \alpha$ and MM1 form stable complexes. MCF10A cell lysates were subjected to IP with anti-p63 or anti-MM1, using normal mouse IgG (MS IgG) or normal rabbit IgG (RB IgG), respectively, as a control. The immunoprecipitates were analyzed by IB with anti-p63 and anti-MM1. Whole cell lysates were used as input controls. Image of either short or long exposure was shown for $\Delta \mathrm{Np} 63 \alpha$ bands. 
based shRNAs. As shown in Figure 4B, knockdown of MM1 led to an increase in expression of Cyclin D1 and CDK4, which are both transcriptional target of c-Myc and are important for cell cycle G1 phase progression [9, 11]; depletion of $\Delta \mathrm{Np} 63 \alpha$ resulted in decreases of Cyclin D1 and CDK4 expressions, which were significantly reversed by simultaneous knockdown of MM1. Further study reveals that this regulation of CDK4 and Cyclin D1 expression mediated by p63 and MM1 occurs at mRNA levels (Figure 4C). These data suggest that endogenous $\mathrm{p} 63 \alpha$ can positively elevate intrinsic c-Myc transactivity via inhibition of MM1. Additionally, simultaneous knockdown of MM1 could rescue, at least in part, down-regulation of c-Myc protein level induced by p63 ablation (Figure 4B). consistent with

A.
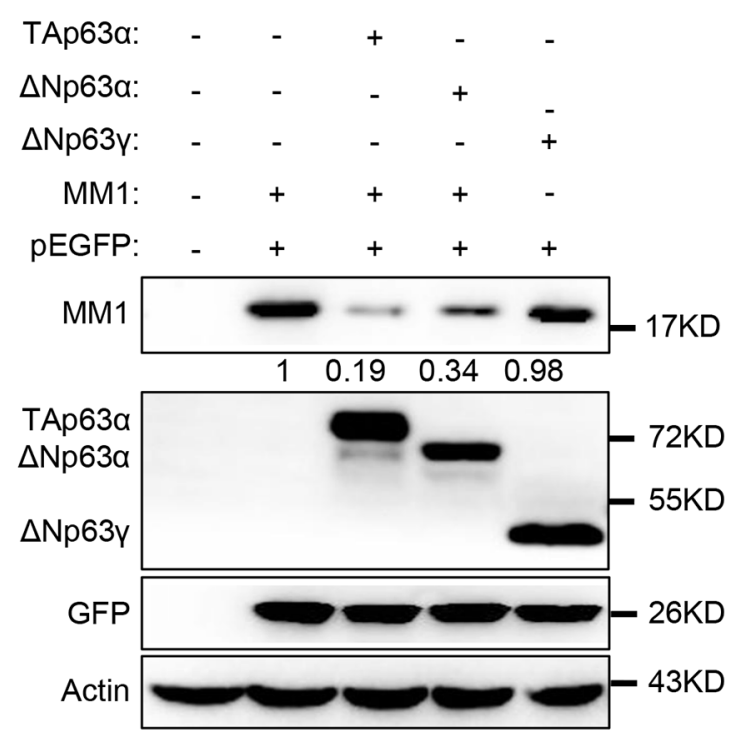

$\mathrm{H} 1299$

B.

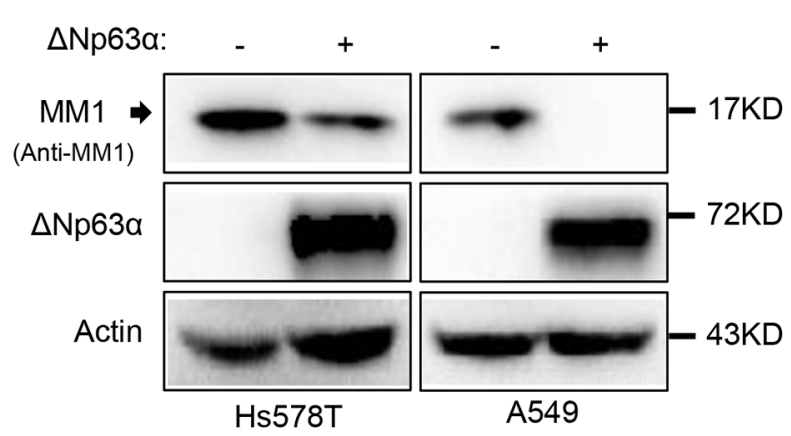

previous reports that $\mathrm{p} 63$ proteins are necessary for basal transcription of c-Myc gene [23], and MM1 may promote Cullin2-mediated degradation of c-Myc protein [29].

Both c-Myc and p63 $\alpha$ are transcription factors involved in cell cycle progression [8, 30-32]. To investigate whether p63 $\alpha$-induced destabilization of MM1 can impact cell cycle, we performed a flow cytometry analysis with MCF10A cells infected with shMM1 or (and) shp63. As shown in Figure 4D, knockdown of p63 $\alpha$ significantly decreased percentage of cells in $\mathrm{S}$ phase, while simultaneous knockdown of $\Delta \mathrm{Np} 63 \alpha$ and MM1 led to cell cycle distribution similar to that of control. This suggests that p63 $\alpha$ can positively regulate cell cycle progression via inhibition of MM1.

C.

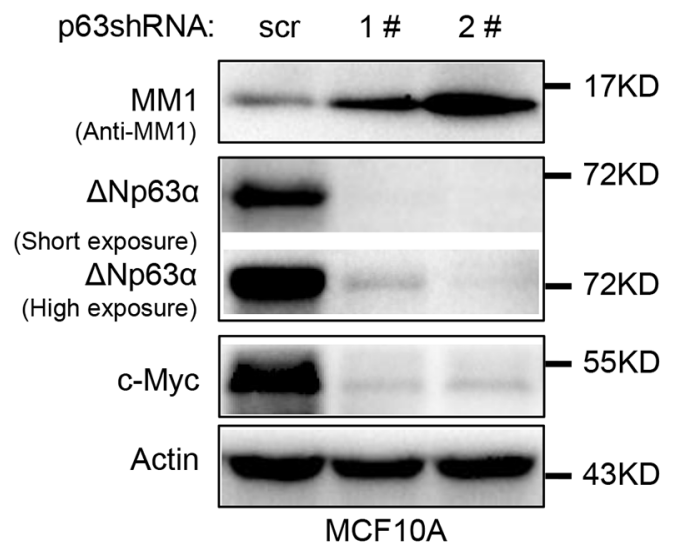

D.

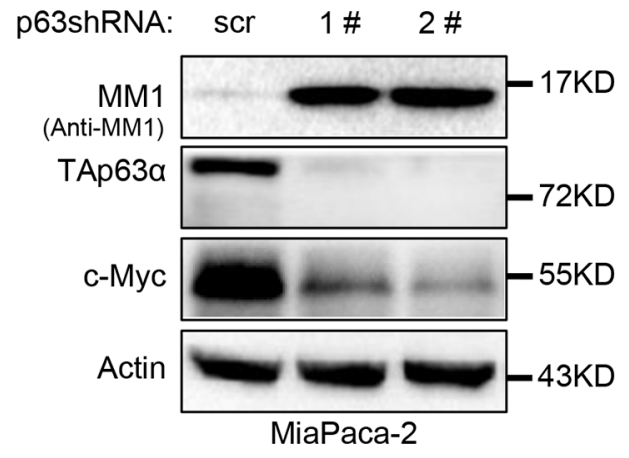

Figure 2: p63 $\alpha$ down-regulates MM1 protein expression. A. $\Delta$ Np63 $\alpha$ down-regulates MM1 expression in H1299 cells. H1299 cells were transfected with indicated expression plasmids. 24 hours post transfection, cells were harvested and subjected to IB analysis for p63, MM1, GFP and Actin. Intensities of MM1 bands were normalized with GFP bands, and that in lane 2 was set as 1. B. $\Delta \mathrm{Np} 63 \alpha$ down-regulates MM1 in Hs578T and A549 cells. Hs578T or A549 cells were infected with lentiviral-based $\Delta \mathrm{Np} 63 \alpha .48$ hours post infection, cells were lysed and subjected to IB analysis for p63, MM1 and Actin. C. and D. Ablation of endogenous $\Delta \mathrm{Np} 63 \alpha$ or TAp63 $\alpha$ up-regulates endogenous MM1. MCF10A or MiaPaCa-2 cells were infected with lentiviral-based shRNA 1\# or 2\#, specific for pan p63, using lentiviral-based scrambled shRNA as a control (scr). 48 hours post infection, cells were lysed and subjected to IB analysis for p63, c-Myc, MM1 and Actin. 
A.

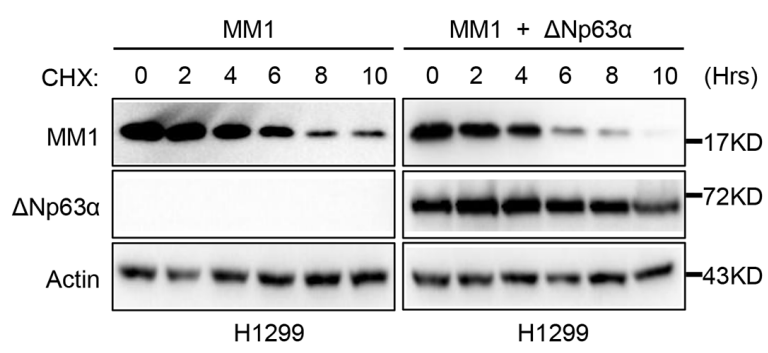

C.

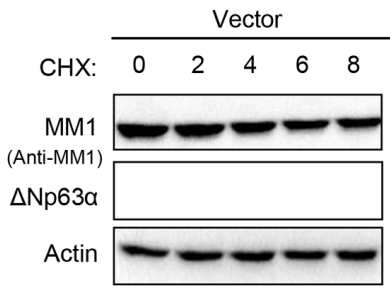

H1299

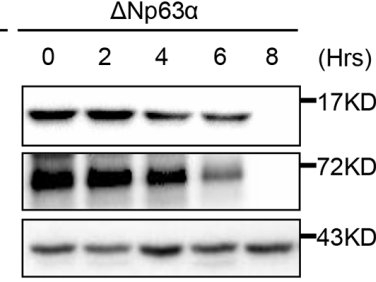

H1299

E.

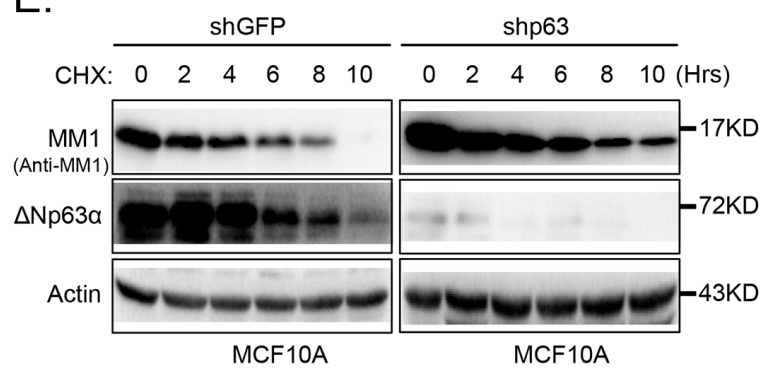

G.
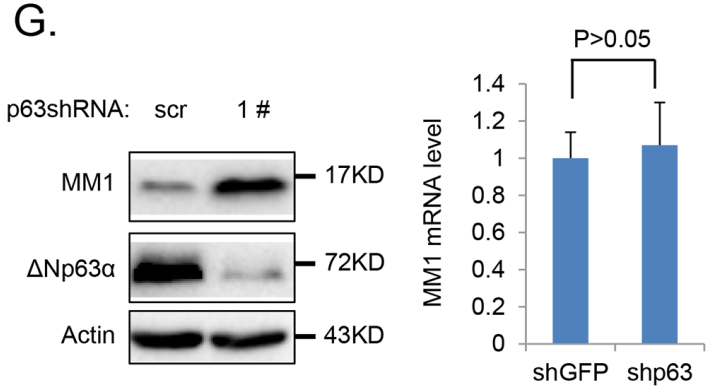

B.

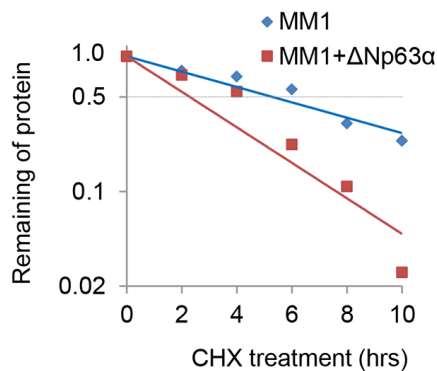

D.

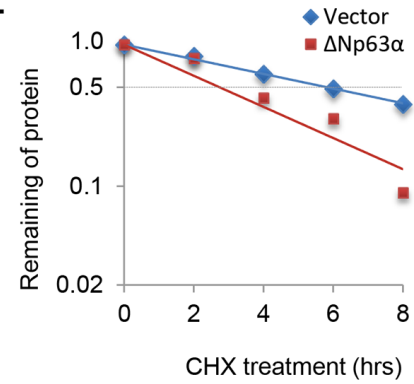

F.

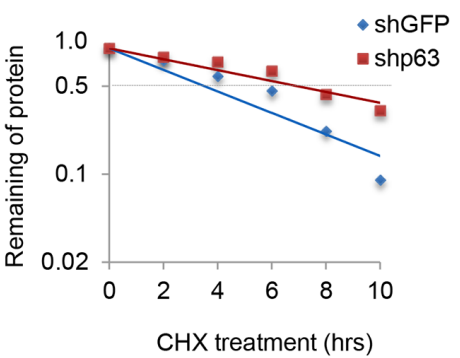

H.

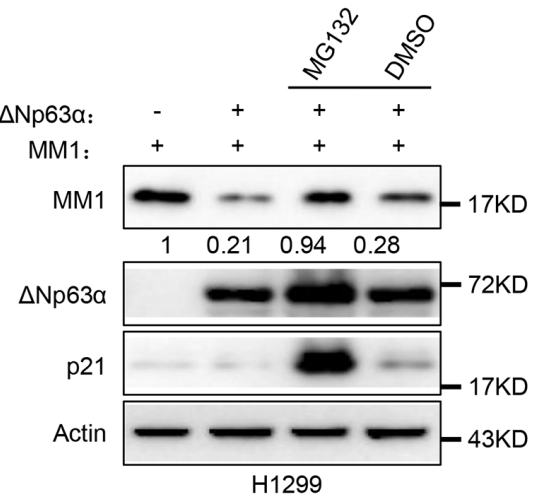

Figure 3: $\Delta \mathrm{Np63 \alpha}$ promotes MM1 proteasome-dependent degradation. A. Ectopic expression of $\Delta \mathrm{Np} 63 \alpha$ shortens half-life of MM1 protein. H1299 cells were co-transfected with FLAG-MM1 together with vector control (FLAG-MM1) or $\triangle$ Np63 $\alpha$ (FLAG-MM1 + $\Delta \mathrm{Np} 63 \alpha$ ). 24 hours post transfection, cells were treated with $100 \mu \mathrm{g} / \mathrm{ml}$ cycloheximide (CHX) for indicated time intervals. Cells were harvested and subjected to IB analysis. B. Quantification results of panel A. Percentages of FLAG-MM1 protein were normalized with Actin, and that for the time point of 0 hour was set as 100. C. and D. Ectopic expression of $\triangle \mathrm{Np} 63 \alpha$ shortens half-life of endogenous MM1 protein. H1299 cells transfected with vector control or $\Delta \mathrm{Np} 63 \alpha$ were subjected to measurement of MM1 protein half-life, using methods abovementioned. E. and $\mathbf{F}$. Depletion of $\triangle$ Np63 $\alpha$ extends half-life of endogenous MM1 protein. MCF10A cells were infected with lentiviral-based shRNA specific for p63 (1\#) to knockdown endogenous $\Delta \mathrm{Np} 63 \alpha$, using lentiviral-based shRNA specific for GFP as a control. 48 hours post infection, cells were lysed and subjected to measurement of MM1 protein half-life. G. Knockdown of endogenous p63 up-regulates endogenous MM1. MCF10A cells were infected with lentiviral-based scrambled or p63 (1\#) shRNAs. 72 hours post infection, cells were subjected to IB or Q-PCR analysis. The Q-PCR data were presented as as means \pm s.e. to measure mRNA levels of MM1, from three independent experiments performed in triplicate. H. Inhibition of proteasome abrogates $\Delta \mathrm{Np} 63 \alpha$-induced down-regulation of MM1. H1299 cells were co-transfected with MM1 plasmid plus vector control or $\Delta \mathrm{Np} 63 \alpha$ plasmid. 24 hours post transfection, cells were treated with MG132 or DMSO for additional 6 hours. Cells were then harvested and subjected to immunoblot analysis. Intensities of MM1 bands were normalized with Actin bands. 


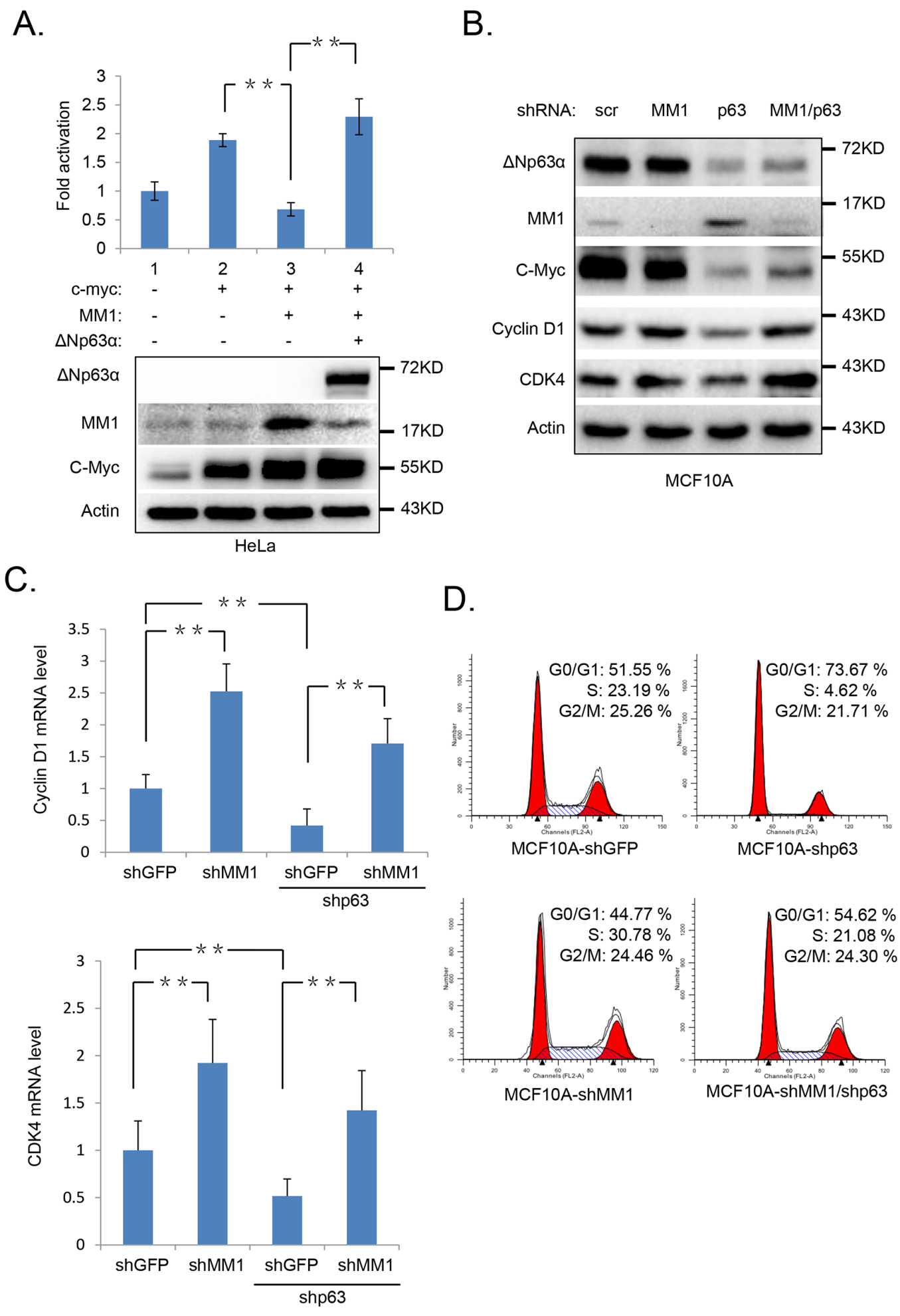

Figure 4: Ablation of $\Delta \mathrm{Np63} \alpha$ down regulates c-Myc transactivity and impair cell cycle progression. A. $\Delta \mathrm{Np} 63 \alpha$ derepresses MM1-mediated suppression of c-Myc transactivity. HeLa cells were co-transfected with MBC1-4-Luc reporter and Renilla plus indicated plasmids. All transfections were performed in triplicates. 48 hours post transfection, cells were lysed and subjected to luciferase assay and IB analysis, respectively. The expression of MBC4-Luc reporter was normalized to Renilla luciferase activity. Results were presented as fold activation of luciferase activity with standard deviation $(\mathrm{n}=3) .{ }^{* *}, \mathrm{p}<0.01$. B-D. Knockdown of $\Delta$ Np63 $\alpha$ down regulates c-Myc and its downstream targets, resulting in cell cycle arrest, which are rescued by shMM1. MCF10A cells were infected with lentiviral-based scrambled, MM1 or (and) p63 (1\#) shRNAs. 72 hours post infection, cells were subjected to IB (B), Q-PCR (C), or FACS (D) analysis. The Q-PCR data were presented as means \pm s.e. to measure mRNA levels of Cyclin D1 and CDK4, from three independent experiments performed in triplicate. ${ }^{* *}, \mathrm{p}<0.01$. 


\section{Correlation of elevation of p63 and c-Myc targets in tumorigenesis}

To explore the clinical relevance of c-Myc activation mediated by $\mathrm{p} 63 \alpha / \mathrm{MM} 1$ pathway, we analyzed Oncomine, an online microarray database. As shown in Figure 5, mRNA levels of p63 and several transcriptional
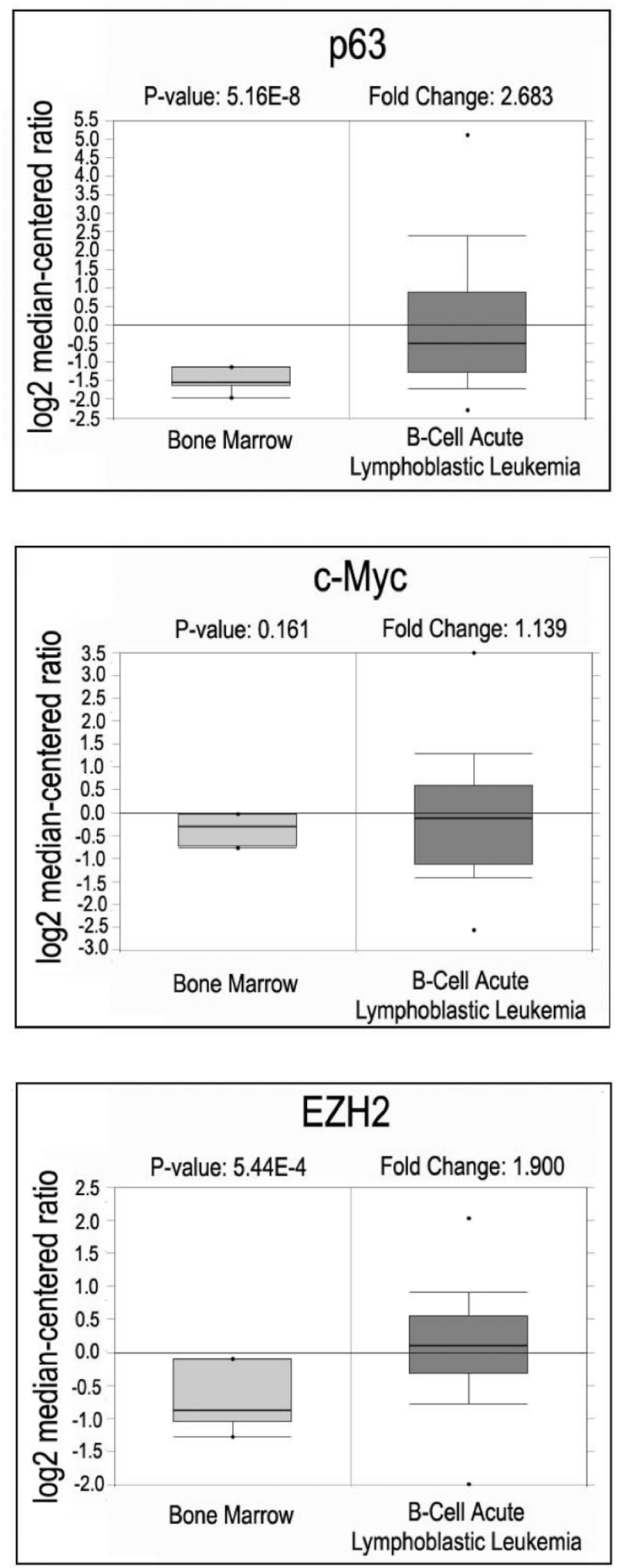

targets of c-Myc, including CDK4, EZH2 and eIF-2 $\alpha$, are concomitantly up-regulated in human B-cell acute lymphoblastic leukemia specimens, while mRNA levels of MM1 and c-Myc remain largely unchanged, suggesting that elevated $\mathrm{p} 63 \alpha$ may stimulate c-Myc transactivity in some cancer types, such as B-cell acute lymphoblastic leukemia.
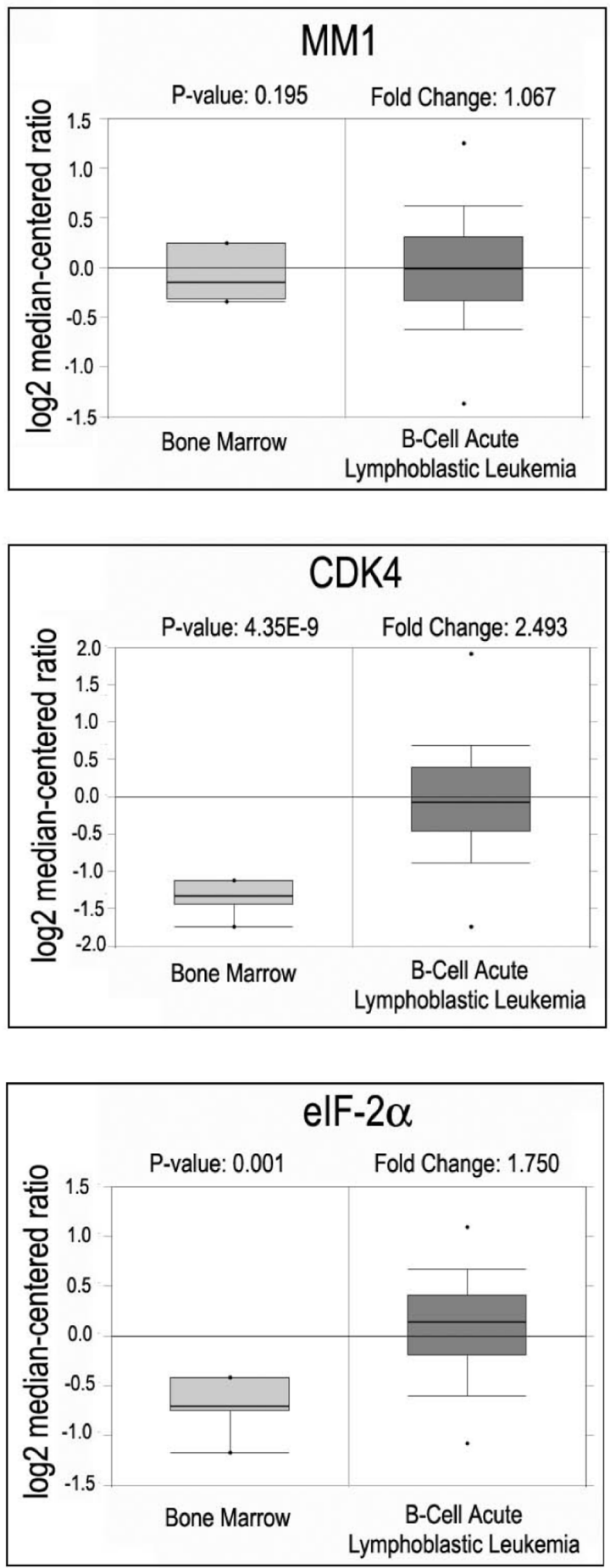

Figure 5: Oncomine analyses of p63, MM1, c-Myc and c-Myc target genes in B-cell acute lymphoblastic leukemia. Box plots representing expression of p63, MM1, c-Myc and its responsive genes from B-cell acute lymphoblastic leukemia samples (Andersson data set). 


\section{DISCUSSION}

p63 and p73 are both members of p53 family. It has been reported that c-Myc inhibitor MM1 binds to $\mathrm{C}$-terminus of p73 $\alpha$ and enhances p $73 \alpha$-dependent transcription [33]. Our present study shows that MM1 associates with C-terminus of p63 $\alpha$, which is homologous to that of $\mathrm{p} 73 \alpha$. We found that $\mathrm{p} 63 \alpha$ physically interacts with MM1 protein and facilitates its proteasomal degradation. Overexpression of p63a effectively shortens half-life of MM1 protein, while knockdown of $\mathrm{p} 63 \alpha$ extends it.

As important transcription factors involved in cell cycle control and tumorigenesis, p63 and c-Myc regulate transcription of their downstream target genes. Recently, it was reported that $\mathrm{p} 63$ is required for basal transcription of c-Myc in human keratinocytes [23]. Our data demonstrate that overexpression of $\Delta \mathrm{Np} 63 \alpha$ can significantly reverse MM1-mediated repression of c-Myc transactivation, consistent with the observation that $\mathrm{p} 63 \alpha$ destabilizes MM1 protein. Furthermore, knockdown of $\Delta \mathrm{Np} 63 \alpha$ in MCF10A cells impairs cell cycle progress, in keeping with our previous report [22]. Importantly, simultaneous knockdown of $\Delta \mathrm{Np} 63 \alpha$ and MM1 effectively restores cell cycle arrest induced by knockdown of $\triangle \mathrm{Np} 63 \alpha$, indicating that MM1 is an important regulator in $\mathrm{p} 63 \alpha$-mediated cell cycle control, likely though modulation of c-Myc activity and function. Moreover, results of clinical analysis indicate that the p63 $\alpha$ /MM1 pathway may derepress c-Myc transactivity in B-cell acute lymphoblastic leukemia.

We speculate that $p 63 \alpha$ can positively affect c-Myc by two different pathways: p63 proteins are necessary for basal transcription of c-Myc in some cell types, possibly via $\mathrm{Wnt} / \beta$-catenin and Notch signaling pathways [23]; on the other hand, p63 $\alpha$ promotes proteasomal degradation of MM1 and consequently derepresses its suppression effects on c-Myc [17, 20, 29]. Taken together, this study reveals a novel way that p63 up-regulates c-Myc activity and function, which may attribute to various biological and pathophysiological processes such as cell cycle and tumorigenesis.

\section{MATERIALS AND METHODS}

\section{Yeast two-hybrid screening}

The cDNA encoding C-terminal region of p63a (amino acid residues 535-680, p63 $\alpha \mathrm{CT}$ ) was cloned into pGBKT7 as a "bait" plasmid. The MATCHMAKER GAL4 Two-Hybrid System 3 (Clontech) and a cDNA library derived from human mammary gland in the pACT2 yeast expression vector (Clontech) were used to screen interacting proteins of $\mathrm{p} 63 \alpha \mathrm{CT}$ according to the manufacturer's instructions.

\section{Cell culture and drug treatment}

H1299, HeLa, 293T, A549, and MiaPaCa-2 cells were maintained in Dulbecco's modified Eagle's medium (DMEM, Hyclone) supplemented with $10 \%$ fetal bovine serum (FBS, Hyclone) and 1\% penicillin/streptomycin (Hyclone). For Hs578T cells, DMEM was supplemented with $10 \%$ FBS plus $10 \mathrm{mg} / \mathrm{ml}$ insulin (Sigma). MCF10A cells were maintained in DMEM/F12 media (Hyclone) supplemented with $20 \mathrm{ng} / \mathrm{ml}$ epidermal growth factor (Invitrogen), $100 \mathrm{ng} / \mathrm{ml}$ cholera toxin (Sigma,), $10 \mathrm{mg} / \mathrm{ml}$ insulin (Sigma), $500 \mathrm{ng} / \mathrm{ml}$ hydrocortisone (Sigma), 1\% penicillin/streptomycin (Hyclone) sulfate and 5\% FBS (Hyclone). All cells were cultured at $37^{\circ} \mathrm{C}$ in a humidified $5 \% \mathrm{CO}_{2}$ incubator. MG132 and cycloheximide (CHX) were purchased from Sigma.

\section{Transfections and immunoblot (IB) analysis}

H1299 or HeLa cells with 50\% confluency in 6-well plates were co-transfected with $400 \mathrm{ng}$ pCMV2-FLAGMM1, 200 ng pEGFP and 400 ng pcDNA3.1/pcDNA3.1-

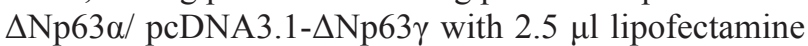
2000 (Invitrogen). 24 hours later, cells were collected, washed with phosphate-buffered saline, and resuspended in EBC250 lysis buffer (50 mM Tris-HCl, pH8.0, $250 \mathrm{mM}$ $\mathrm{NaCl}, 0.5 \%$ Nonidet P-40, $0.2 \mathrm{mM}$ phenylmethylsulfonyl fluoride, $2 \mu \mathrm{g} / \mathrm{ml}$ leupeptin, $2 \mu \mathrm{g} / \mathrm{ml}$ aprotinin, $50 \mathrm{mM}$ $\mathrm{NaF}$, and $0.5 \mathrm{mM} \mathrm{Na}_{3} \mathrm{VO}_{4}$ ). Protein concentration was determined using the Bio-Rad protein assay reagent (Bio-Rad). An equal amount of protein (about $50 \mu \mathrm{g}$ total protein) was loaded, separated on a $10 \%$ SDS-PAGE, transferred to polyvinylidene difluoride membrane (BioRad), and hybridized to an appropriate primary antibody and horseradish peroxidase-conjugated secondary antibody for subsequent detection with ECL (Millipore). IB analysis was performed with anti-p63 (Santa Cruz, sc8431, 1:200), anti-FLAG (Sigma, F1804, 1:1000), antiMM1 (Epitomics, 5689-1, 1:500), anti-c-Myc (Epitomics, 1472-1, 1:1000), anti-GFP (Santa Cruz, sc-9996, 1:1000), anti-p21 (Abcam, ab54562, 1:500), anti-Cyclin D1 (Epitomics, 2261-1, 1:1000), anti-CDK4 (Santa Cruz, sc260, 1:1000), or anti-Actin (Abcam, ab13772, 1:1000).

\section{Co-immunoprecipitation (Co-IP)}

H1299 cells co-transfected with indicated plasmids or MCF10A cells were lysed in ice-cold EBC250 lysis buffer for $30 \mathrm{~min}$ on ice. Lysates were cleared by centrifugation at $16,000 \mathrm{~g}$ for $15 \mathrm{~min}$. Protein concentration was determined using the Bio-Rad protein assay reagent (Bio-Rad). Cell lysates containing $2 \mathrm{mg}$ total protein were incubated with $2 \mu \mathrm{g}$ anti-FLAG (Sigma, F1804), Myc tag antibody (Abcam, ab32), p63 antibody (Santa Cruz, sc-8431), or anti-MM1 (Epitomics, 5689- 
1), respectively, at $4^{\circ} \mathrm{C}$ for 2 hours. Then the immune complexes were precipitated with protein A-agarose at $4^{\circ} \mathrm{C}$ for 3 hours The immunoprecipitates were washed with EBC250 lysis buffer, separated by SDS-PAGE, and subjected to IB analysis with indicated antibodies.

\section{Quantitative PCR (Q-PCR) analysis}

Total RNA was isolated using RNeasy Mini Kit (Qiagen), followed by reverse transcription using OneStep RT-PCR kit (Qiagen) according to themanufacturers' instruction. Q-PCR was performed using QuantiTect SYBR Green PCR Kit (Qiagen) according to the manufacturer's instructions. Primer sequences were described in Supplementary Table 1. All primers spanned at least one intron and control amplification was performed on RNA samples not subjected to the reverse transcription, in parallel, to ensure no contaminating genomic DNA was present.

\section{Lentiviral infection}

$293 \mathrm{~T}$ cells were cotransfected with $\mathrm{pLVX}-\Delta \mathrm{Np} 63 \alpha /$ pLKO.1-p63shRNA/ pLKO.1-MM1shRNA along with psPAX2 and pMD2.G lentiviral packaging plasmids by Lipofectamine 2000. The medium were collected and filtered through a $0.45 \mu \mathrm{M}$ filter to remove debris 48 hours later. The lentiviral particles were then concentrated by ultra-centrifugation $\left(20,000 \mathrm{rpm}, 2 \mathrm{~h}\right.$ at $\left.4^{\circ} \mathrm{C}\right)$, resuspended in fresh medium at $4^{\circ} \mathrm{C}$ for $24 \mathrm{~h}$, then supplemented with polybrene $(10 \mu \mathrm{g} / \mathrm{ml})$ and used to infect A549, Hs578T, MCF10A, or MiaPaCa-2 cells. 48 hours after infection, the cells were subjected to IB analysis or selected in growth medium supplemented with $2 \mu \mathrm{g} / \mathrm{ml}$ puromycin for two days.

\section{Protein stability assay}

Cells were transiently transfected (or infected) with indicated plasmids or shRNA lentiviruses. 24 hours post transfection (or infection), cells were treated with Cycloheximide $(\mathrm{CHX})$ at a final concentration of 100 $\mu \mathrm{g} / \mathrm{ml}$ for $0 \sim 10$ more hours. Cells were collected at the indicated time points. Equal amounts of total proteins were subjected to IB analysis. Intensities of bands for MM1 or Actin were quantified with Image Lab (BioRad). The protein levels of MM1 were normalized with Actin, and that of the time point of 0 hour was set as 100 percent.

\section{Assays for proteasome-dependent MM1 Protein degradation}

H1299 cells with 50\% confluency in 6-well plates were co-transfected with $800 \mathrm{ng}$ pCMV2-FlagMM1 and $800 \mathrm{ng}$ pcDNA3.1 or pcDNA3.1- $\Delta$ Np63 $\alpha$ by lipofectamine 2000. 24 hours later, cells were treated with $20 \mu \mathrm{M}$ MG132 or equal amount of solvent vehicle DMSO for 6 more hours. Then cells were collected and lysed with EBC250. Equal amounts of total protein were subjected to western for p63, p21, MM1, and Actin.

\section{Luciferase assays}

HeLa cells were plated for transfection at a density of $2 \times 10^{5}$ cells/well in 12-well plates for 24 hour. Cells were co-transfected with $100 \mathrm{ng}$ MBC1-4-Luc reporter and 5 ng pRL-TK Renilla plasmids, plus c-Myc and (or) FLAG-MM1, $\Delta$ Np63 $\alpha$ expression plasmids (200 ng each). The total amount of DNA was kept constant (705 ng per transfection) with pcDNA3.1 vector. Cells were harvested at 48 hours after transfection and lysed in Passive Lysis Buffer (Promega). Lysates were analyzed for firefly and Renilla luciferase activities using the Dual Luciferase Reagent Assay Kit (Promega). Luciferase assays were performed as described (Luciferase assay system; Promega). Luminescence was measured in a luminometer. Relative luciferase activity was determined by normalizing luciferase activity with Renilla.

\section{Flow cytometry (FACS) analysis}

Cells were fixed in $70 \%$ ethanol at $4{ }^{\circ} \mathrm{C}$ overnight and stained with $50 \mu \mathrm{g} / \mathrm{ml}$ propidium iodide (Sigma) supplemented with $100 \mu \mathrm{g} / \mathrm{ml}$ RNase A (Sigma) for $40 \mathrm{~min}$ at $37^{\circ} \mathrm{C}$ in the dark. Then cells were subjected to FCM analysis by FACScan flow cytometer (Becton Dickson). Data were analyzed using the Cell Quest program.

\section{Bioinformatic and statistical analysis}

Oncomine (Compendia Bioscience, Ann Arbor, MI, USA) was used for bioinformatic analysis of gene expression. Quantitative data were analyzed statistically using Student's $t$-test to assess significance. Data are presented as means \pm s.e., as noted in figure legends.

\section{ACKNOWLEDGMENTS}

This work was supported by National Basic Research Program of China (\#2012CB910700 to ZX Xiao), National Natural Science Foundation of China (31171362 and 81520108020 to ZX Xiao) and Science and Technology Department of Sichuan Province (2016JY0152 to C Li).

\section{CONFLICTS OF INTEREST}

The authors declare no conflicts of interest to this work. 


\section{REFERENCES}

1. Yang A, Schweitzer R, Sun D, Kaghad M, Walker N, Bronson RT, Tabin C, Sharpe A, Caput D, Crum C, McKeon F. p63 is essential for regenerative proliferation in limb, craniofacial and epithelial development. Nature. 1999; 398:714-718.

2. Yang A, Kaghad M, Wang Y, Gillett E, Fleming MD, Dotsch V, Andrews NC, Caput D, McKeon F. p63, a p53 homolog at 3q27-29, encodes multiple products with transactivating, death-inducing, and dominant-negative activities. Mol Cell. 1998; 2:305-316.

3. Mangiulli M, Valletti A, Caratozzolo MF, Tullo A, Sbisa E, Pesole G, D'Erchia AM. Identification and functional characterization of two new transcriptional variants of the human p63 gene. Nucleic Acids Res. 2009; 37:6092-6104.

4. Thanos CD, Bowie JU. p53 Family members p63 and p73 are SAM domain-containing proteins. Protein Sci. 1999; 8:1708-1710.

5. Li C, Xiao ZX. Regulation of p63 protein stability via ubiquitin-proteasome pathway. Biomed Res Int. 2014; 2014:175721.

6. Perez CA, Pietenpol JA. Transcriptional programs regulated by p63 in normal epithelium and tumors. Cell Cycle. 2007; 6:246-254.

7. Nesbit CE, Tersak JM, Prochownik EV. MYC oncogenes and human neoplastic disease. Oncogene. 1999; 18:3004-3016.

8. Dang CV. c-Myc target genes involved in cell growth, apoptosis, and metabolism. Mol Cell Biol. 1999; 19:1-11.

9. Hermeking H, Rago C, Schuhmacher M, Li Q, Barrett JF, Obaya AJ, O'Connell BC, Mateyak MK, Tam W, Kohlhuber F, Dang CV, Sedivy JM, Eick D, Vogelstein B, Kinzler KW. Identification of CDK4 as a target of c-MYC. Proc Natl Acad Sci U S A. 2000; 97:2229-2234.

10. Miliani de Marval PL, Macias E, Rounbehler R, Sicinski P, Kiyokawa H, Johnson DG, Conti CJ, Rodriguez-Puebla ML. Lack of cyclin-dependent kinase 4 inhibits c-myc tumorigenic activities in epithelial tissues. Mol Cell Biol. 2004; 24:7538-7547.

11. Yang H, Li TW, Ko KS, Xia M, Lu SC. Switch from MntMax to Myc-Max induces p53 and cyclin D1 expression and apoptosis during cholestasis in mouse and human hepatocytes. Hepatology 2009; 49:860-870.

12. Koh CM, Iwata T, Zheng Q, Bethel C, Yegnasubramanian $\mathrm{S}$, De Marzo AM. Myc enforces overexpression of EZH2 in early prostatic neoplasia via transcriptional and post-transcriptional mechanisms. Oncotarget. 2011; 2:669-683. doi: 10.18632/oncotarget.327.

13. Benvenisty N, Leder A, Kuo A, Leder P. An embryonically expressed gene is a target for c-Myc regulation via the c-Myc-binding sequence. Genes Dev. 1992; 6:2513-2523.

14. Schuldiner O, Eden A, Ben-Yosef T, Yanuka O, Simchen G, Benvenisty N. ECA39, a conserved gene regulated by c-Myc in mice, is involved in G1/S cell cycle regulation in yeast. Proc Natl Acad Sci U S A. 1996; 93:7143-7148.

15. Rosenwald IB, Rhoads DB, Callanan LD, Isselbacher KJ, Schmidt EV. Increased expression of eukaryotic translation initiation factors eIF-4E and eIF-2 alpha in response to growth induction by c-myc. Proc Natl Acad Sci U S A. 1993; 90:6175-6178.

16. Galaktionov K, Chen X, Beach D. Cdc25 cell-cycle phosphatase as a target of c-myc. Nature. 1996; 382:511-517.

17. Mori K, Maeda Y, Kitaura H, Taira T, Iguchi-Ariga SM, Ariga H. MM-1, a novel c-Myc-associating protein that represses transcriptional activity of c-Myc. J Biol Chem. 1998; 273:29794-29800.

18. Vainberg IE, Lewis SA, Rommelaere H, Ampe C, Vandekerckhove J, Klein HL, Cowan NJ. Prefoldin, a chaperone that delivers unfolded proteins to cytosolic chaperonin. Cell. 1998; 93:863-873.

19. Sakamuro D, Prendergast GC. New Myc-interacting proteins: a second Myc network emerges. Oncogene. 1999; 18:2942-2954.

20. Fujioka Y, Taira T, Maeda Y, Tanaka S, Nishihara H, Iguchi-Ariga SM, Nagashima K, Ariga H. MM-1, a c-Mycbinding protein, is a candidate for a tumor suppressor in leukemia/lymphoma and tongue cancer. J Biol Chem. 2001; 276:45137-45144.

21. Straub WE, Weber TA, Schafer B, Candi E, Durst F, Ou HD, Rajalingam K, Melino G, Dotsch V. The C-terminus of p63 contains multiple regulatory elements with different functions. Cell Death Dis. 2010; 1:e5.

22. Li C, Chang DL, Yang Z, Qi J, Liu R, He H, Li D, Xiao ZX. Pin1 modulates p63alpha protein stability in regulation of cell survival, proliferation and tumor formation. Cell Death Dis. 2013; 4:e943.

23. $\mathrm{Wu} \mathrm{N}$, Rollin J, Masse I, Lamartine J, Gidrol X. p63 regulates human keratinocyte proliferation via MYCregulated gene network and differentiation commitment through cell adhesion-related gene network. J Biol Chem. 2012; 287:5627-5638.

24. Xu E, Zhang J, Zhang M, Jiang Y, Cho SJ, Chen X. RNAbinding protein RBM24 regulates p63 expression via mRNA stability. Mol Cancer Res. 2014; 12:359-369.

25. Kibbe MR, Nie S, Seol DW, Kovesdi I, Lizonova A, Makaroun M, Billiar TR, Tzeng E. Nitric oxide prevents p21 degradation with the ubiquitin-proteasome pathway in vascular smooth muscle cells. J Vasc Surg. 2000; 31:364-374.

26. Luscher B, Eisenman RN. New light on Myc and Myb. Part I. Myc. Genes Dev. 1990; 4:2025-2035. 
27. Blackwood EM, Eisenman RN. Max: a helix-loophelix zipper protein that forms a sequence-specific DNA-binding complex with Myc. Science. 1991; 251:1211-1217.

28. Blackwood EM, Luscher B, Eisenman RN. Myc and Max associate in vivo. Genes Dev. 1992; 6:71-80.

29. Kimura Y, Nagao A, Fujioka Y, Satou A, Taira T, IguchiAriga SM, Ariga H. MM-1 facilitates degradation of c-Myc by recruiting proteasome and a novel ubiquitin E3 ligase. Int J Oncol. 2007; 31:829-836.

30. Dohn M, Zhang S, Chen X. p63alpha and DeltaNp63alpha can induce cell cycle arrest and apoptosis and differentially regulate p53 target genes. Oncogene. 2001; 20:3193-3205.

31. Dang CV. MYC on the path to cancer. Cell. 2012; 149:22-35.

32. Dang CV, O'Donnell KA, Zeller KI, Nguyen T, Osthus RC, Li F. The c-Myc target gene network. Semin Cancer Biol. 2006; 16:253-264.

33. Watanabe K, Ozaki T, Nakagawa T, Miyazaki K, Takahashi M, Hosoda M, Hayashi S, Todo S, Nakagawara A. Physical interaction of $\mathrm{p} 73$ with c-Myc and MM1, a c-Myc-binding protein, and modulation of the p73 function. J Biol Chem. 2002; 277:15113-15123. 